\title{
Static magnetic field enhances synthesis and secretion of membrane-derived microvesicles (MVs) rich in VEGF and BMP-2 in equine adipose-derived stromal cells (EqASCs) - a new approach in veterinary regenerative medicine
}

\author{
Monika Marędziak • Krzysztof Marycz • Daniel \\ Lewandowski • Anna Siudzińska • Agnieszka Śmieszek
}

Received: 12 July 2014 / Accepted: 23 September 2014 /Published online: 27 November 2014 / Editor: T. Okamoto

(C) Society for In Vitro Biology 2014

\begin{abstract}
The aim of this work study was to evaluate the cytophysiological activity of equine adipose-derived stem cells (ASCs) cultured under conditions of static magnetic field. Investigated cells were exposed to a static magnetic field (MF) with the intensity of $0.5 \mathrm{~T}$. In order to investigate the effects of magnetic field on stem cell signaling, the localization and density and content of microvesicles (MVs) as well as morphology, ultrastructure, and proliferation rate of equine ASCs were evaluated. Results showed that potential of equine adipose-derived mesenchymal stem cells was accelerated when magnetic field was applied. Resazurin-based assay indicated that the cells cultured in the magnetic field reached the population doubling time earlier and colony-forming potential of equine ASCs was higher when cells were cultured under magnetic field conditions. Morphological and ultrastructural examination of equine ASCs showed that the exposure to magnetic field did not cause any significant changes in cell morphology whereas the polarity of the cells was observed under the magnetic field conditions in ultrastructural
\end{abstract}

M. Marędziak $(\bowtie) \cdot$ K. Marycz $\cdot$ A. Siudzińska $\cdot$ A. Śmieszek Electron Microscopy Laboratory, University of Environmental and Life Sciences Wroclaw, Kozuchowska 5b, 51-631 Wroclaw, Poland e-mail: monika.maredziak@gmail.com

M. Marędziak

Department of Animal Physiology and Biostructure, Faculty of Veterinary Medicine, Wrocław University of Environmental and Life Sciences, Wroclaw, Poland

K. Marycz $\cdot$ A. Śmieszek

Wroclaw Research Centre EIT+, Stablowicka 147, 54-066 Wroclaw, Poland

D. Lewandowski

Institute of Materials Science and Applied Mechanics, Wroclaw University of Technology, Smoluchowskiego 25, 50-372 Wroclaw, Poland examinations. Exposition to MF resulted in a considerable increase in the number of secreted MVs - we have clearly observed the differences between the numbers of MVs shed from the cells cultured under MF in comparison to the control culture and were rich in growth factors. Microvesicles derived from ASCs cultured in the MF condition might be utilized in the stem cell-based treatment of equine musculoskeletal disorders and tendon injuries.

Keywords Magnetic field · Adipose-derived mesenchymal stem cells $\cdot$ Microvesicles $\cdot$ Equine

\section{Introduction}

The essential components of the current veterinary regenerative medicine are the therapies involving application of multipotent stromal cells (MSCs). These cells have the capacity to self-renew and differentiate into the cells of various lineages, e.g., osteoblasts, chondrocytes, or adipocytes, but they can also express markers specific for endothelial cells and cardiomyocytes (Marion and Mao 2006; Gimble et al. 2007; Augello and De Bari 2010). They are characterized by multipotency, plasticity, and immunomodulatory properties, which make them a promising tool in the modern veterinary regenerative medicine (Mizuno et al. 2012; Zomorodian and Eslaminejad 2012). Bone marrow and adipose tissue provide the most accessible source of adult stem cells. Moreover, the role of adipose-derived stem cells (ASCs) is becoming increasingly important particularly in the veterinary clinical treatment of large animals (Guest et al. 2008; Marycz et al. 2012a; Barba et al. 2013). Recently, MSCs have been more readily applied in equine veterinary medicine. The results of many research groups as well as our previous studies have 
confirmed the positive clinical effect of MSC transplantations in equine musculoskeletal disorders, involving, e.g., tendons or cartilages (Del Bue et al. 2008; Marycz et al. 2012b; Nicpoń et al. 2013).

There is a growing body of evidence that a key role in accelerating the regeneration of damaged tissue involves the improvement of the cell-to-cell contact through MSC transplantations. Previous studies suggest that the intercellular signaling is an essential part of the interaction process between the cells and can be mediated by the transfer of secreted molecules (Wang and Lu 2006; Liu et al. 2009; Fierabracci et al. 2013; Turturici et al. 2014). It has been shown that two types of membrane-derived vesicles are released from the mesenchymal cells, i.e., exosomes and microvesicles (MVs). Although the release of exosomes has been known for many years, the fact that the cells are able to shed other types of vesicles from their plasma membrane has been recognized only recently. Whereas exosomes are small $(50-100 \mathrm{~nm})$ membranous vesicles predominantly originating from preformed multivesicular bodies released upon fusion with plasma membrane, microvesicles $(100-1,000 \mathrm{~nm})$ are arising from the budding of the plasma membrane through the reorganization of membrane phospholipids and increased concentration of $\mathrm{Ca}^{2+}$ that activates enzymatic cascade (MuralidharanChari et al. 2010; Inal et al. 2013; Turturici et al. 2014). Microvesicles secreted by MSCs can travel certain distances, and thus transfer the bioactive molecules to remote sites. When interacting with the microvesicles, the cells are able to take up their content and utilize it for biological activity. As previously described, a wide range of molecules, including cytokines, growth factors, as well as miRNA have been identified in MVs derived from MSCs (more than 700 proteins and 150 miRNAs). Indeed, MVs are known to promote the regeneration of a number of tissues (e.g., kidney, heart, liver, or nervous tissues) by exerting an effect on target cells or inducing neighboring cells by the transfer of cytokines, such as transforming growth factor, hepatocyte growth factor, or vascular endothelial growth factor (ExoCarta database contains a comprehensive list of proteins, lipids, and RNAs associated with MVs <http://www.exocarta.org $>$ ) (Camussi et al. 2010; Biancone et al. 2012; Mathivanan et al. 2012).

Given these facts, the acceleration of secretion of MVs from multipotent stromal cells of adipose origin (ASCs) seems to be a crucial factor in the improvement of their regenerative potential. The magnetic field (MF) is one of many factors that could be successfully applied to support cytophysiological activity of equine ASCs. In our previous studies, we have shown that MF improved proliferation activity of equine stem cells; thus, we wanted to investigate whether this factor can also improve secretory activity of equine ASCs (Marędziak et al. 2014). The biological effects of static MFs have been a subject of research for many years. It is well known that MF play a role in the repair of tissues and is commonly applied in the context of clinical application (Markov 2007; Colbert et al. 2009; Esposito et al. 2013; Pesce et al. 2013). The magnetic field has been used for over $30 \mathrm{yr}$ to enhance the healing process and treatment of patients with orthopedic diseases. Magnetotherapy is regarded as a safe, non-invasive, and effective method (Rosen 2003; Ganesan et al. 2009). Numerous studies have shown that MF can affect various cell functions, such as proliferation, differentiation, gene expression, inhibition of apoptosis, and reduction of proinflammatory cytokines (Landry et al. 1997; Heermeier et al. 1998; De Mattei et al. 1999; Rosen 2003; Grassi et al. 2004). Stem cell therapy combined with MF treatment may provide promising outcomes in the clinical practice, resulting in an effective reduction of pain and inflammation, and what is more, improvement of the regeneration process.

The aim of the present study was to evaluate the cytophysiological activity of equine ASCs cultured under conditions of static magnetic field. In order to investigate the effects of magnetic field on stem cell signaling, we have evaluated the localization and density of microvesicles as well as morphology, ultrastructure, and proliferation rate of equine ASCs. We also determined the content of microvesicles performed with ELISA assays. To our knowledge, this is the first comprehensive report providing information on the upregulation of ASC regenerative potential and basic mechanisms responsible for microvesicle-mediated tissue regeneration.

\section{Materials and Methods}

Ethical approval. This work was approved by the Second Local Bioethics Committee at the Department of Biology and Animal Breeding, Wroclaw University of Environmental and Life Sciences, Wroclaw, Chelmonskiego 38C, Poland (177/2010 of 15 November 2010). The experimental protocols were carried out with the additional permission of the animals' owners. Fat tissue (2 g) for the study was collected from a donor horse from the tail base area. Adipose tissue biopsies were performed under local anesthesia.

Isolation of ASCs. Cells were isolated using modified methods previously described by Grzesiak et al. (2011) Briefly, the adipose tissue was washed extensively with Hank's Balanced Salt Solution (HBSS) containing 1\% antibioticantimicotic solution (penicillin/streptomycin/amphotericin b) to remove blood vessel contamination. Then the tissues were cut into small pieces and dissociated with collagenase type I $(1 \mathrm{mg} / \mathrm{mL})$ for $40 \mathrm{~min}$. The sample was centrifuged at $1,200 \times \mathrm{g}$ for $10 \mathrm{~min}$. The supernatant was discarded and the cell pellet was resuspended in growth media. The cell suspension was then added to the cell culture flask. 
Isolation and collection of MVs. Equine ASCs were centrifuged at $2,000 \times \mathrm{g}$ for $15 \mathrm{~min}$ at $4^{\circ} \mathrm{C}$, and the MV-enriched supernatants were collected and filtered using PureExo ${ }^{\circledR}$ (101Bio, Palo Alto, CA). The protein content in EqASCderived MVs was evaluated using Bradford assay as described by Majka et al. (2007).

Immunophenotyping. The characterization of equine MSCs was performed by immunophenotyping using surface markers. The expression of CD29, CD44, CD73b, CD105, and CD45 antigens in equine adipose-derived MSCs was analyzed using primary antibodies (all purchased from Sigma-Aldrich, Munich, Germany, except for anti-CD44 obtained from R\&D, Abingdon, UK) and fluorescent microscopy. Briefly, cells were fixed in 4\% PFA (10 min) and then incubated in $1 \% \mathrm{BSA} / 10 \%$ normal goat serum $/ 0.3 \mathrm{M}$ glycine in $0.1 \%$ PBS-Tween for $1 \mathrm{~h}$ to permeabilize the cells and block nonspecific protein-protein interactions. The cells were then incubated overnight with antibodies (1:1,000 dilutions) at $4^{\circ} \mathrm{C}$. Alexa Fluor 488 goat anti-rabbit (green) was used as the secondary antibody at a 1:1,000 dilution for $1 \mathrm{~h}$. DAPI was used to stain the cell nuclei at a concentration of 1:1,000.

Cell culture. All stages of the procedure were conducted under aseptic, standardized maintenance and culture conditions $\left(37^{\circ} \mathrm{C}, 5 \% \mathrm{CO}_{2}\right)$. DMEM/Ham's F12 medium was applied in the primary culture complemented with $15 \%$ fetal bovine serum (FBS) and 1\% antibiotic-antimycotic solution, while DMEM with $4,500 \mathrm{mg} / \mathrm{L}$ of glucose containing $10 \%$ FBS and $1 \%$ antibiotic-antimycotic solution was used in the secondary cultures.

The primary seeded cells were allowed to adhere to the flask for $24 \mathrm{~h}$ before changing the medium, and the medium was changed every $2 \mathrm{~d}$ thereafter. The cells were passaged using trypsin solution (TrypLE ${ }^{\mathrm{TM}}$; Life Technologies, Paisley, UK) when they reached about $80 \%$ confluence. For assay, the cells were transferred into six-well plates, suspended in $2 \mathrm{~mL}$ of medium at a concentration of $30 \times 10^{3}$ cells per well.

Magnetic field exposure. Static MF was produced by a single neodymium magnet (Nd-Fe-B) type N38 of a cylindrical shape and a diameter of $5 \mathrm{~mm}$ and a length of $20 \mathrm{~mm}$ of known intensity, placed under each culture well. The intensity of the magnetic field generated was checked using a single axis Hall sensor and was measured for three axes. The total magnetic field intensity was calculated using the results of a measurement of the three vector components according the following formula:

$B_{C}=\sqrt{B_{x}^{2}+B_{y}^{2}+B_{z}^{2}}$

where $B_{x}, B_{y}$ and $B_{z}$ are individual component values for the $x$, $y$, and $z$ axis, respectively.
Morphology and cell activity evaluation. The morphology of the cells and nuclei distribution were evaluated after $7 \mathrm{~d}$ of the experiment, under fluorescent and scanning electron microscope (SEM). Additionally, SEM analysis allowed comparing the quantity of secreted MVs. In order to perform observations, the cells were washed in HBSS and sequentially fixed with $4 \%$ ice-cold paraformaldehyde (30 $\mathrm{min}$ ) and $0.1 \%$ Triton X-100 (15 min). After fixation and permeabilization, the cells were washed and stained with atto-488-labeled phalloidin. Samples were incubated at room temperature in the dark for $30 \mathrm{~min}$, and subsequently counterstained with DAPI for $5 \mathrm{~min}$. After triple washing, the cells were observed under inverted epifluorescence microscope (Axio Observer A.1, Zeiss, Göttingen, Germany). For scanning electron microscopy, the cells were washed with distilled water and dehydrated in a graded ethanol series. The cells were observed using a SE1 detector, at $10 \mathrm{kV}$ filament tension (SEM, Zeiss Evo LS 15).

Examination of ASC ultrastructure. All steps of the procedure were followed by centrifugation at $1,200 \times g$ for $5 \mathrm{~min}$. The cells were collected and fixed with $2.5 \%$ gluteralaldehyde for $24 \mathrm{~h}$ at $4^{\circ} \mathrm{C}$. Then the cells were washed three times with distilled water and incubated for $2 \mathrm{~h}$ with $1 \%$ osmium tetroxide. After this time, the cells were washed twice with distilled water. The samples were counterstained with lead citrate and uranyl acetate ( 1 and $1.5 \mathrm{~h}$ incubation, respectively), dehydrated in a graded series of acetone, and embedded in an Agar Low Viscosity Resin Kit (Agar Scientific Ltd, Essex, UK).

Prior to sectioning, the cells were incubated $48 \mathrm{~h}$ at $60^{\circ} \mathrm{C}$ for polymerization. The samples were sectioned into ultrathin slices $(70 \mathrm{~nm})$ and collected on copper grids. Observations were carried using STEM detector at $10 \mathrm{kV}$ filament tension.

Estimation of the size and location of MVs. The activity of cells manifested by the secretion of MVs was analyzed using computer-assisted image analyses with a Java-based open source image processing software ImageJ v1.48c. Image analysis was performed on six randomly chosen fields of previously captured SEM images. The analysis included measurements of active areas secreting the MVs and the cell count. In brief, to expose and evaluate active area of the culture surface, SEM images were transformed into 3D surface plots. Parameters of the picture were adjusted so that the active area was colored in orange, while non-active in blue and violet. 3D color plots were subsequently converted into two-dimensional images and adjusted with a color threshold plugin. The selected active area was measured using a Measure plugin, excluding the surface area of the nuclei, and expressed as a percentage of the cell surface in the image. The total number of microvesicles on a particular image was counted using a Cell Counter plugin and recalculated into the number of vesicles per cell. 
Cell viability and colony-forming efficiency assay. The number of viable cells in the control and samples cultured in the presence of magnetic field was determined by resazurin-based assay kit (Alamar Blue) and performed according to the manufacturer's instructions $(T O X-8)$. The viability of cells was estimated after $1,3,5$, and $7 \mathrm{~d}$.

For assay, the culture medium was removed and replaced with the medium containing $10 \%$ of resazurin dye. Cells were incubated with dye solution for $2 \mathrm{~h}$ at $37^{\circ} \mathrm{C}$, after which the supernatants were collected. Dye reduction was determined spectrophotometrically at a wavelength of $600 \mathrm{~nm}$ and a reference wavelength of $690 \mathrm{~nm}$ using a microplate reader (Spectrostar Nano, BMG Labtech, Ortenberg, Germany). Proliferation activity of the cells was determined as the population doubling time (PDT) calculated according to the method described previously (Marycz et al. 2013) and supported by the online software (Roth 2006).

Colony-forming efficiency (CFE) assay was performed by counting and seeding equine ASCs after three passages into six-well plates. The cell density was equal to 100 cells per well and cells were cultured in a normal DMEM medium with 4,500 g glucose, both in the presence of MF and in the control conditions at $37^{\circ} \mathrm{C}$. The medium was removed after $7 \mathrm{~d}$ of culture and cells were washed twice with HBSS. Next, the cells were stained with a crystal violet solution for $10 \mathrm{~min}$ at room temperature. Colonies with a cell count greater than 50 were counted and CFE was defined according to the formula described previously by Chen et al. (2009):

$\mathrm{CFE}=\frac{\text { number of colonies }}{\text { initial cell number }} \times 100 \%$

The experiment was performed in triplicate.

Analyses of the MVs contents. ELISA assays were performed to quantitatively analyze the levels of BMP-2, VEGF, TNF- $\alpha$, and p53 in MVs using supernatants collected from 7-d equine ASC cultures stimulated with MF and cultured in control conditions. All ELISA kits were purchased from MyBiosource Inc, San Diego, CA, except for TNF- $\alpha$, which was purchased from R\&D systems. The reactions were performed according to the manufacturer's instructions.

Analyses of calcium concentration in MVs were carried out by scanning electron microscopy combined with energy dispersive X-ray analysis (SEM/EDX). The Quantax detector (Brüker, Billerica, MA) with $10 \mathrm{kV}$ filament tension was used in the spot analysis of six randomly selected MVs. The values obtained were presented in weight percentage ( $\mathrm{wt} \%$ ).

All reagents were purchased from Sigma-Aldrich unless specified otherwise.

\section{Results}

MSC immunophenotyping and morphology. The homogenous population of equine MSCs derived from adipose tissue was obtained after three passages. Specific immunofluorescence staining confirmed the expression of CD29, CD44, CD73b, and CD105 markers, while the hematopoietic lineage marker, CD45, was excluded (Fig. 1).

Morphological examination of equine ASCs revealed that the exposure to magnetic field did not cause any significant changes in cell morphology. The cells exposed to magnetic field exhibited morphological features similar to those of the control cells, maintaining typical fibroblast-like morphology. The cells formed a homogenous monolayer of the bipolar spindle-like cells with a regular pattern (Fig. 2). DAPI staining of the nuclei indicated that the cells cultured in the presence of a magnetic field had asymmetrically located nuclei, translocated to one of the cell poles (Fig. 2A), whereas in the cells cultured in the control conditions, the nuclei were positioned centrally (Fig. 2B). Cells cultured in the presence of MF adhered evenly to the surface of the well and did not cluster. Cells that were not exposed to MF densely covered the central part of the well and closely adhered to each other.

Ultrastructure of ASCs. The influence of the magnetic field on the ultrastructure of ASCs was investigated using transmission electronic microscopy (Fig. $2 C, D$ ). The polarity of the cells was observed under the magnetic field conditions, which was manifested by the displacement of the nuclei to the peripheral part of the cells (Fig. 2C). The cytoplasm of the cells exposed to magnetic field contained abundant and welldeveloped organelles, including mitochondria, Golgi bodies, and rough endoplasmic reticulum arranged in the vicinity of the nuclei area. Thus, it was difficult to clearly determine the symmetry of the cells. In the MF environment, the cells had larger nuclei with loose chromatin and the number of mitochondria was noticeably higher (Fig. 2C). A pleat-like plasma membrane invagination was clearly visible in the cells from samples cultured in the magnetic field. Moreover, a large number of vesicles were observed shedding from the cell membrane. In the control samples, cell nuclei were positioned centrally and the organelles were evenly distributed in the cytoplasm. Single vesicles secreted from the plasma membrane were also detected (Fig. 2D).

Evaluation of $M V$ density and arrangement. Exposition to MF resulted in a considerable increase in the number of secreted MVs (size of 100-1,000 nm) in equine ASCs. These observations were confirmed by electron microscopy as well as by computer-assisted method using a Java-based image processing software ImageJ v1.48. Although in the majority of electronographs microvesicles were located around the nuclei (Figs. $3 A, B$ and $4 A-D$ ), the wide spectrum of MVs 

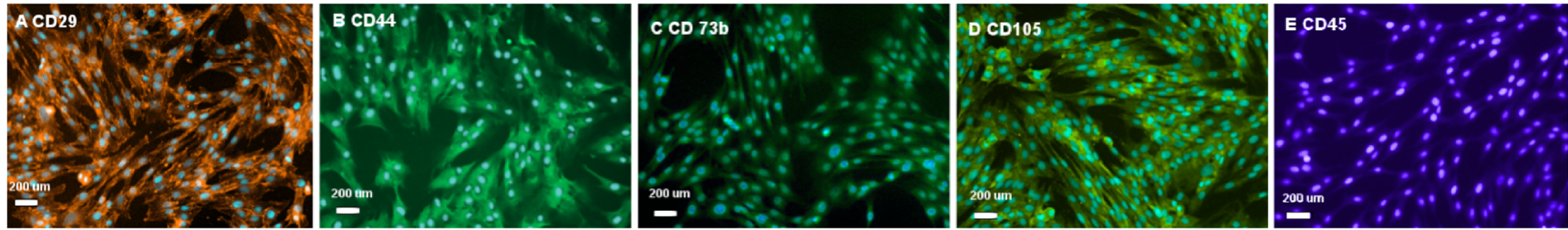

Figure 1. Analysis of ASC phenotypes using specific immunofluorescence staining. The population obtained expressed the following markers: CD29 $(A)$, CD73b (B), CD105 (C), CD44 (D), CD $45(E)$. Appropriate scale bars are indicated in each panel.

was also observed at the border area of cells (Fig. $4 C, D$ ). Measurements of MVs diameters demonstrated the presence of particles of size greater than $100 \mathrm{~nm}$, which were predominant in the samples analyzed (Fig. 3C,D). MVs greater than $100 \mathrm{~nm}$ were significantly more common in the cells exposed to MF in comparison to control (Figs. $4 A-D$ and $5 G-I$ ). Since significant differences in the number of microvesicles have been attributed to major changes in physiological activity, we assessed the number of MVs per cell as the active cell area (Fig. 4). The percentage of MV area was determined using ImageJ software, and it amounted to about $20 \%$ in the samples exposed to MF. In the control samples, the percentage of the active area reached $11 \%$ (Fig. 4G, $H$ ).

ImageJ 3D images clearly demonstrated a higher activity of the cells. Red elevated points on the images visibly presented thicker areas on the cell surface indicating vesicles secreted from the plasma.

In addition, equine ASCs with numerous MVs present were characterized by the formation of cytonemes and nanotubes (Fig. 3A). These structures are considered important mediators of cell-to-cell communication. The shedding of MVs and development of cytonemes improve the contact between cells.

Population doubling time and colony-forming efficiency of equine ASCs in the magnetic field. The proliferation assay analysis showed that the magnetic field conditions contributed significantly to the change in PDT (Fig. 5A). The results of resazurin-based assay indicated that the cells cultured in the magnetic field reached the population doubling time earlier (by $5 \%$ ) than the cells cultured in the normal conditions. Population doubling times for ASCs cultured under magnetic field and in the control conditions amounted to 53.4 and $56.1 \mathrm{~h}$, respectively.

Single cell-derived colonies formed from ASCs plated at low density. The colony-forming efficiency was different for cells cultured under the magnetic field conditions. It was found that the MF caused an increase in the number of colonies greater than 50 cells (Fig. $5 B$ ). In control conditions, the number of colonies formed from 100 cells was approximately $47 \%$ lower.
Figure 2. Comparison of equine ASC morphology nuclei distribution $(A, B)$ and ultrastructure $(C, D)$ after exposure to a magnetic field $(A, C)$ and under control conditions $(B, D)$. Red asterisks indicate displacement of the nuclei to the peripheral part of the cells. Appropriate scale bars are indicated in each panel.
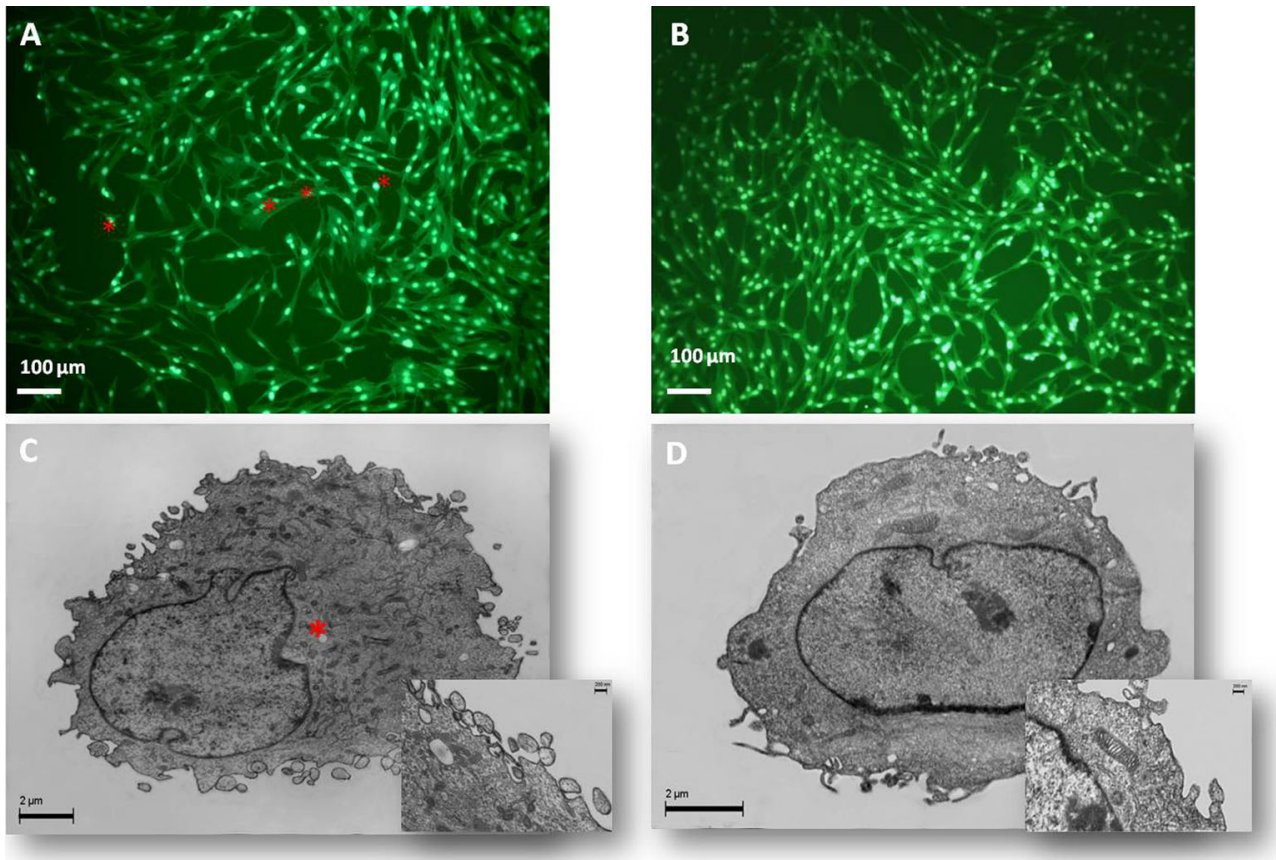

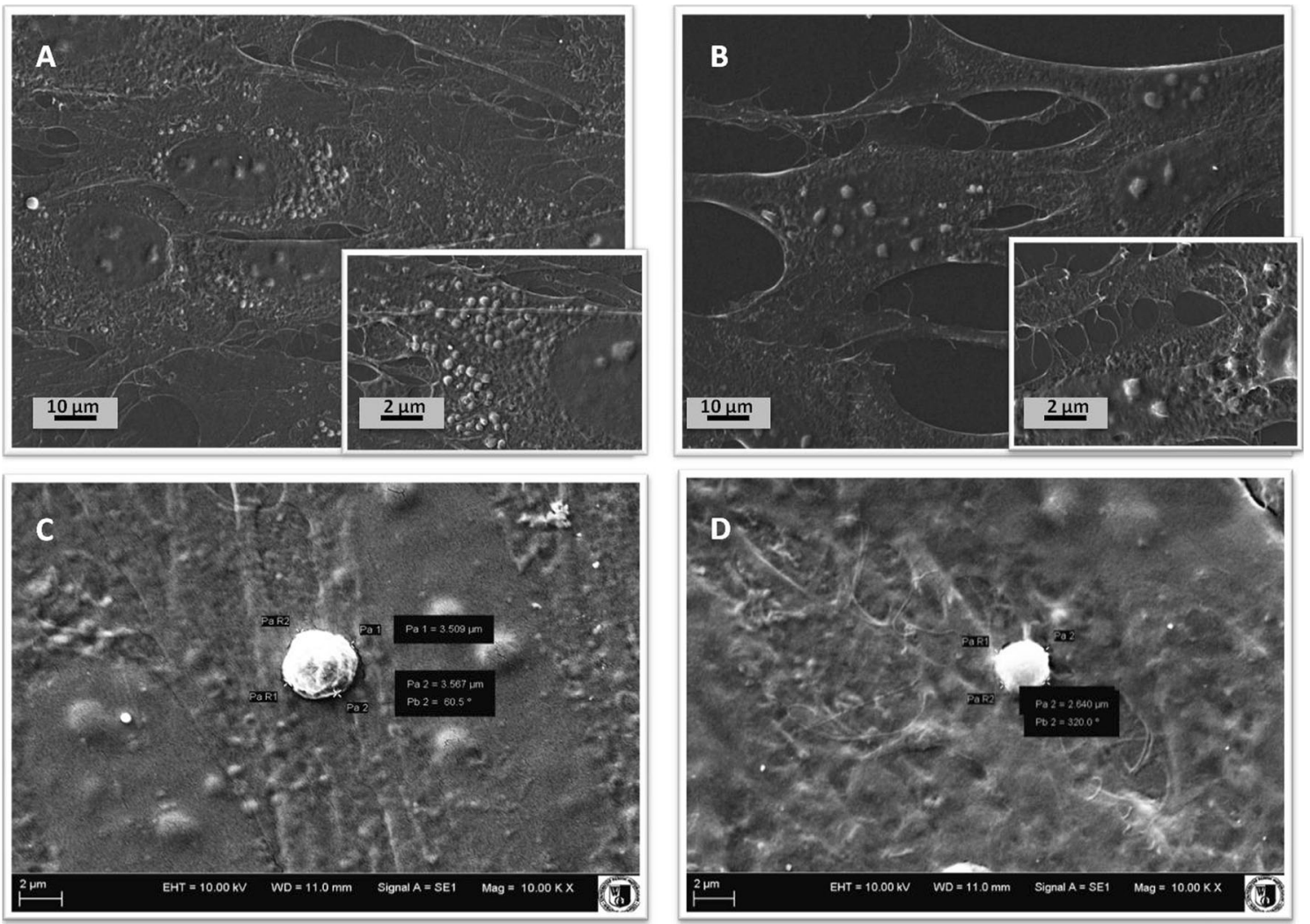

Figure 3. SEM images of equine ASC cultures exposed to magnetic field $(A)$ and the control culture $(B)$; MVs secreted from the cells cultured under magnetic field $(C)$ and control conditions $(D)$. MVs diameters and appropriate scale bars are indicated.

The content of $M V s$. In vitro kinetics of BMP-2, VEGF, $\mathrm{p} 53$, and TNF- $\alpha$ was evaluated using ELISA quantitative tests. Figure $6 A, B$ shows that the release of both investigated growth factors (VEGF and BMP-2) was significantly higher in the MVs derived from the cells treated with the magnetic field compared to the control samples. The concentration of BMP-2 was $327( \pm 73) \mathrm{ng} / \mathrm{mL}$ in the magnetic field-treated samples, whereas the concentration of BMP-2 in the control conditions was equal to 280 ( \pm 67 ) (Fig. 6A). A similar trend was observed when VEGF concentration was investigated. MVs derived from MF-treated cells had higher levels of VEGF $(1,445( \pm 25) n g / L)$, while in the control MVs this concentration was significantly lower $(1,309( \pm 55) \mathrm{ng} / \mathrm{L})$ (Fig. 6B). A similar correlation was observed when a concentration of p53 was investigated in the MVs derived from cells cultured in the MF.

The level of p53 was higher in the MVs from the cells treated with $\mathrm{MF}(503 \pm 51)$ in comparison to the control (446 \pm 33) (Fig. 6D). Interestingly, a downregulation of TNF- $\alpha$ secretion was observed after the MF treatment, as the concentration of the cytokine examined amounted to $59 \pm 1.53 \mathrm{pg} /$ $\mathrm{mL}$, while the control MVs contained $63.5 \pm 1.06 \mathrm{pg} / \mathrm{mL}$ of TNF- $\alpha$ (Fig. $6 C$ ).

Energy dispersive X-ray spectrometer (EDX) analysis of the calcium concentration in the MVs indicated a lower content of this element in the samples cultured under MF conditions (Fig. $6 F, G$ ) in comparison to the control samples (Fig. 6E, $G$ ).

\section{Discussion}

Stem cells have been utilized in a number of studies, and over the last decade, progress has been achieved both at the level of in vitro as well as in vivo preclinical research. It has been well documented that the application of adipose-derived mesenchymal stromal cells in the therapy of equine musculoskeletal and tendon disorders causes a significant decrease in the recurrence rate in animals treated with cells $(27 \%)$ compared to those treated conventionally (56\%) (Godwin et al. 2012; Carvalho et al. 2013). The positive effects of MSC transplantation have been demonstrated and they are associated with their ability to release mediators responsible for the cell therapeutic effects (Baglio et al. 2012; Sabin and Kikyo 2014). The most important features of stem cells described thus far is their paracrine action on neighboring cells and a consequent improvement of cell-to-cell contact. The exchange of information between stem cells and cells from the injured tissue is reciprocal (Biancone et al. 2012; Raisi et al. 2014). 

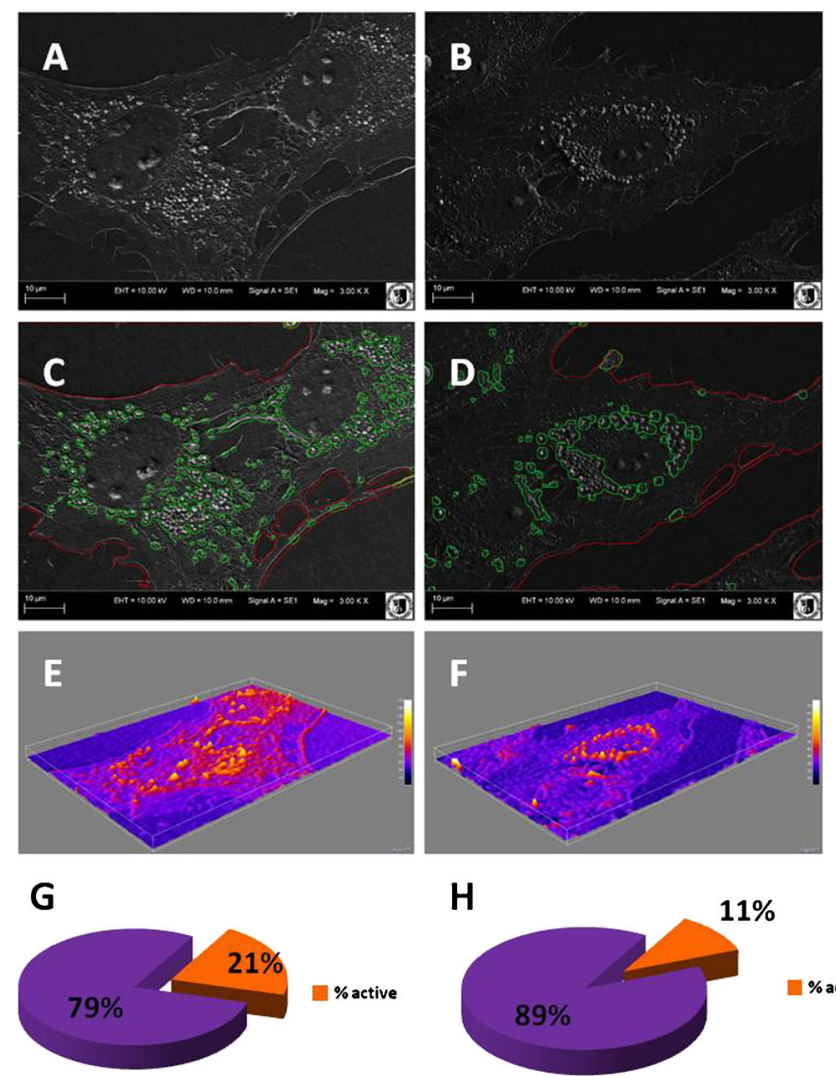

H

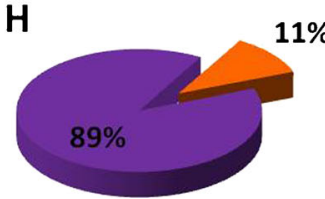

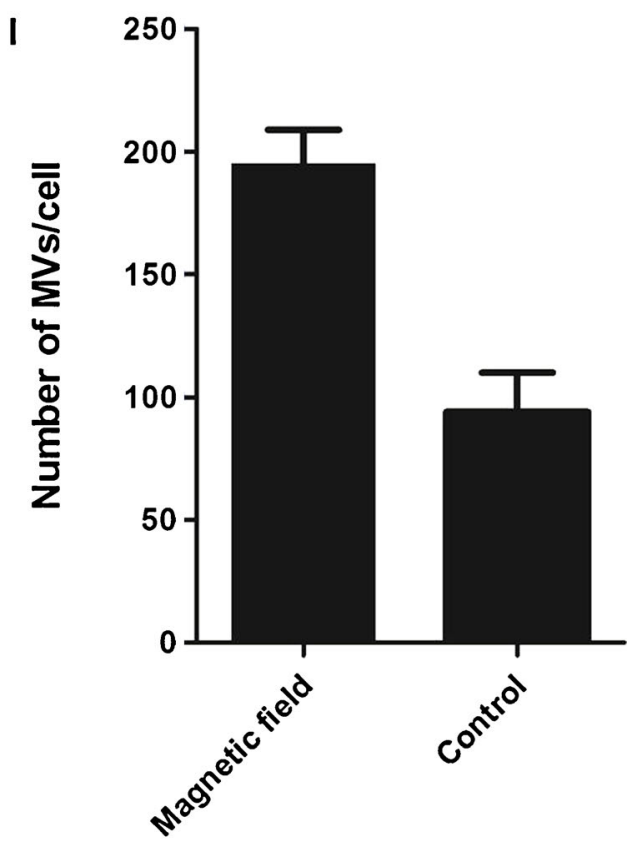

Figure 4. Comparison of the number of MVs on the cell surface after exposure to magnetic field $(A, C, E, G)$ and control conditions $(B, D, F$, $H$ ). In the figure panels $C$ and $D$, specific indication was used to

visualized MVs (green dots) and boundaries of cells (red line). The total number of microvesicles per cell- differences between cells cultured in the presence of MF and in control conditions $(I)$.

Microvesicles secreted from adult stromal cells have numerous biological functions that facilitate the maintenance of stem cells properties. Evidence has accumulated that the amount and content of MVs consistently vary depending on the

microenvironmental conditions, and particularly when cells are subjected to stress factors (Deregibus et al. 2010; Ratajczak 2011). The mechanism of releasing MVs is thought to be part of the normal physiology of cells. However, cell

\section{Population doubling time}

A

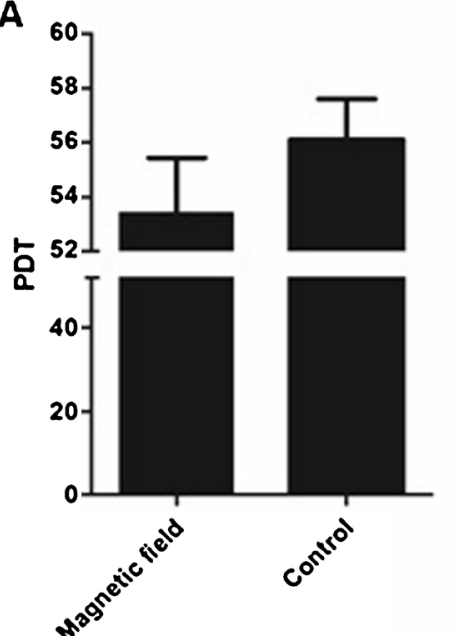

CFE-assay
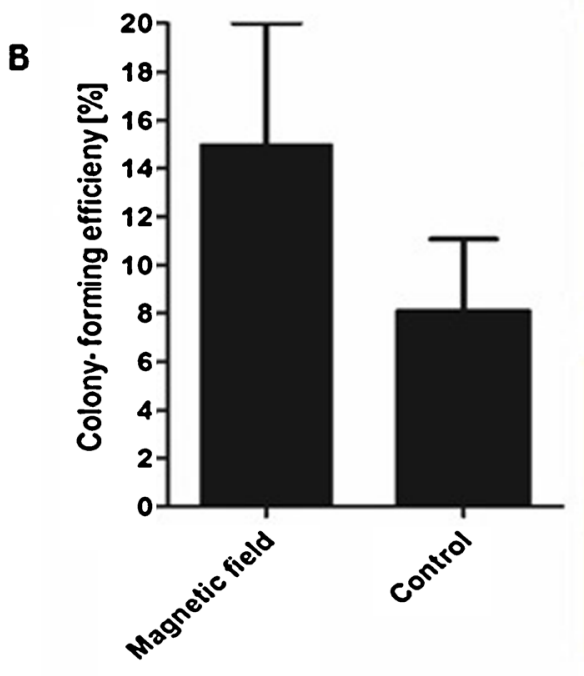
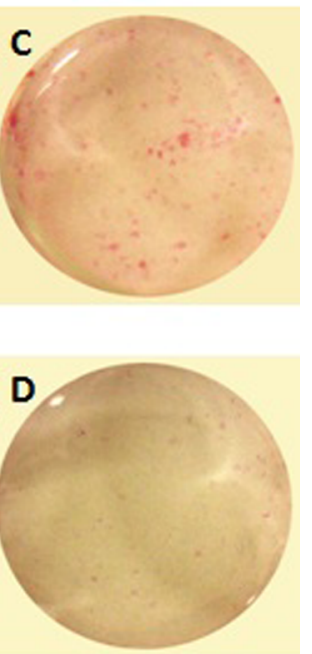

Figure 5. Population doubling time of equine ASCs cultured in control conditions and in the presence of magnetic field $(A)$. Analysis of colony-forming efficiency $(B, C, D)$ under $\mathrm{MF}(B, C)$ and control conditions $(B, D)$. 

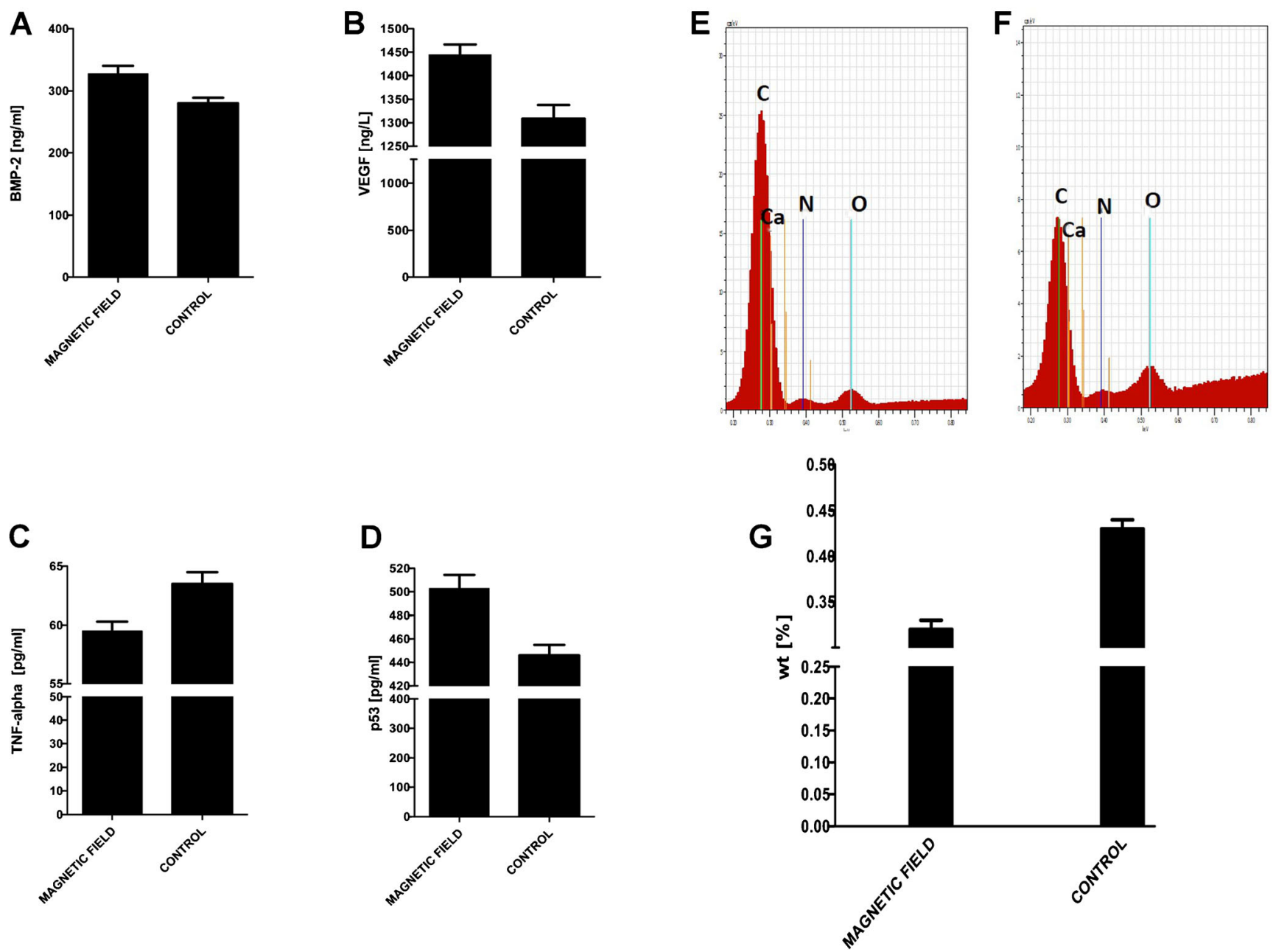

Figure 6. Quantitative ELISA results of BMP-2 $(A), \operatorname{VEGF}(B), \mathrm{TNF}-\alpha(C)$, and p53 content in MVs from magnetic field and control conditions. EDX analysis of the elements content in samples after magnetic field treatment $(E)$ and control $(F)$ in particular calcium concentration $(G)$.

activation leading to changes in the cytoskeleton results in a loss of membrane asymmetry and membrane blebbing, and consequent MV formation and release from the plasma membrane (Inal et al. 2013; Raposo and Stoorvogel 2013). Various factors can affect the cell shape and alter the properties of plasma membrane, including secretory characteristics, which in turn may improve cellular communication and enhance regenerative potential. Kotani et al. (2002) demonstrated that MF exerts an effect on cells by changing their orientation and polarity. Those findings strongly correlates with our observations, as we have found that nuclei migrate to one of the cell poles under MF conditions, which leads to an asymmetrical location of organelles in the cell bodies. Other research groups suggested that MF can influence the orientation of macromolecules (i.e., collagen), thus the cell polarity and spatial distribution of cellular organelles (Torbet and Ronzière 1984). Finally, cells tend to release various biologically active factors, e.g., membrane-derived vesicles (MVs) or exosomes (Ex) in a specific direction. Moreover, it was assumed that the organelles under magnetic field are translocated to a specific pole of the cell, thereby establishing new polarity leading to the restructuring of the tissue. The magnetic field acts on the tissue by linking vesicles with the signaling function, translocation of organelles to the new areas of the cell, and converting tissue geometry. In our research, we have clearly observed the differences between the number of MVs shed from the cells cultured under MF in comparison to the control culture (Fig. 4A). It proved that the static magnetic field improved the activity of stromal stem cells. Moreover, the presence of numerous cytonemes in EqASCs cultured in MF additionally confirmed these assumptions.

In the current study, we have investigated whether static magnetic field can improve the activity of equine ASCs, and in consequence enhance the release of microvesicles rich in growth factors, which have been shown to be crucial for the regeneration of musculoskeletal system and tendons. We have observed that EqASCs under MF conditions showed higher secretory activity. These findings closely correlated with the analysis of the cell proliferation factor and the ability of a 
single cell to form a colony. EqASCs cultured in the MF had a lower population doubling time, which indicated that the MF can positively influence the proliferative potential. This effect was consistent with our previous studies, where we observed a higher proliferative activity of adipose-derived mesenchymal stem cell cultured under MF conditions (Marędziak et al. 2014). The increased proliferation of the cells is connected with their higher secretory activity. The production of membrane-derived vesicles is often associated with the reaction to cell activation or their membrane depolarization. Influx and export of $\mathrm{Ca}^{2+}$ are the mechanisms that control the formation and secretion of MVs. Calcium ions contribute to cytoskeleton reorganization through the activation of cytosolic proteases that cut the proteins responsible for membrane budding. Exposition to the magnetic field leads to an increase of the number of receptors responsible for $\mathrm{Ca}^{2+}$ ion influx and cell phosphorylation (Ikehara et al. 1998; Stratton et al. 2013). It has been suggested that the F-actin components of microfilaments may interrelate their polyelectrolyte properties with the magnetic field, allowing calcium ion conductance into the cytosol (Gartzke and Lange 2002). The results presented by Stratton et al. (2013) suggested that the MF accelerated cell membrane activity, allowing calcium influx, which in turn initiated the release of microvesicles from stimulated cells. Based on our SEM/EDX results, we could clearly demonstrate that the activation of calcium-dependent channels was critical for the vesicle formation and release. Our experiments showed that the content of $\mathrm{Ca}^{2+}$ ions was lower in the samples cultured under MF conditions. The lower concentration of $\mathrm{Ca}^{2+}$ in microvesicles derived from MF cultures proved that these ions are involved in membrane reorganization and are not secreted with MVs. It is also well known that both investigated factors, MVs and MF, are involved in regeneration. Magnetic field was shown to act as an induction factor, while MVs are involved in the transfer of secreted factors. Qiu et al. (2007) have demonstrated that the static MF induced the expression of BMP-2 in rat calvarian cells, which enhanced the osteogenesis. In addition, the high concentration of BMP-2 was found in the plasma of fibroblasts, osteoblasts, cementocytes, and odontoblasts after the MF treatment (Qiu et al. 2004, 2005). Bone morphogenic protein has been reported in many studies as a key player in bone as well as cartilage regeneration. Our research is in good agreement with the above findings, as we observed a significantly higher level of BMP-2 in MVs derived from EqASCs cultured under MF conditions. The magnetic field has been also reported to influence not only the osteogenic properties of the cells but also to improve the process of vascularization. In the present study, we have detected the higher concentration of VEGF in MVs under MF conditions. Thus, we conclude that MF may enhance vascularization, which is considered an essential factor in the regeneration process. Our results are also consistent with the findings of Okano et al. (2006) who showed that the MF stimulation of vascular endothelial cells resulted mainly in the promotion or enhancement of arteriogenesis. The magnetic field acts on two levels - accelerating the release of BMP-2 and VEGF from the cells and at the same time enhancing the secretion of MVs and transfer of these proteins from cell to cell, which can improve the regeneration process. What is more, we have noticed an increased concentration of $\mathrm{p} 53$ in the MVs derived from EqASC cultures where MF was applied. The study of ArmesillaDiaz et al. (2009) showed that the presence of p53 was necessary to maintain a stable culture of MSCs. Deficiency of $\mathrm{p} 53$ could lead to altered MSC differentiation, suggesting that $\mathrm{p} 53$ might play a regulatory role in this process. In addition, a lower concentration of TNF- $\alpha$ was observed at the same time. It is well documented that both MF and MVs possess anti-inflammatory properties (Biancone et al. 2012; Ross and Harrison 2013; Vergallo et al. 2013; Raisi et al. 2014). Inhibition of TNF- $\alpha$ secretion by MF and lack of its cytotoxic effect seem to be advantageous in the treatment of inflammatory diseases, which is associated with an increase in the number of activated cells.

\section{Conclusions}

The results obtained confirmed that the magnetic field exposure caused an enhanced cytophysiological activity of the equine ASCs in the culture. Our findings indicate that the MF can directly stimulate and promote the secretion of membrane-derived microvesicles in the equine adipose-derived mesenchymal stem cells. What is more, our findings clearly support the thesis that the combination of magnetic field with stem cell therapy might be used as a factor controlling cell orientation and improving cell-cell communication. Our results suggest that the microvesicles derived from ASCs cultured in the MF condition are rich in specific growth factors and might be potentially applied in the treatment of equine musculoskeletal disorders and tendon injuries.

Acknowledgments The research was supported by Wroclaw Research Centre EIT+ under the project "Biotechnologies and advanced medical technologies"-BioMed (POIG.01.01.02-02-003/08) financed from the European Regional Development Fund (Operational Programmed Innovative Economy, 1.1.2.).

\section{References}

Armesilla-Diaz A, Elvira G, Silva A (2009) p53 regulates the proliferation, differentiation and spontaneous transformation of mesenchymal stem cells. Exp Cell Res 315(20):3598-3610

Augello A, De Bari C (2010) The regulation of differentiation in mesenchymal stem cells. Hum Gene Ther 21(10):1226-1238 
Baglio SR, Pegtel DM, Baldini N (2012) Mesenchymal stem cell secreted vesicles provide novel opportunities in (stem) cell-free therapy. Front Physiol 3:359

Barba M, Cicione C, Bernardini C, Michetti F, Lattanzi W (2013) Adipose-derived mesenchymal cells for bone regeneration: state of the art. Biomed Res Int 2013:416391

Biancone L, Bruno S, Deregibus MC, Tetta C, Camussi G (2012) Therapeutic potential of mesenchymal stem cell-derived microvesicles. Nephrol Dial Transplant 27(8):3037-3042

Camussi G, Deregibus MC, Bruno S, Cantaluppi V, Biancone L (2010) Exosomes/microvesicles as a mechanism of cell-to-cell communication. Kidney Int 78(9):838-848

Carvalho AD, Badial PR, Alvarez LE, Yamada AL, Borges AS, Deffune E, Hussni CA, Garcia Alves AL (2013) Equine tendonitis therapy using mesenchymal stem cells and platelet concentrates: a randomized controlled trial. Stem Cell Res Ther 4(4):85

Chen T, Zhou Y, Tan WS (2009) Influence of lactic acid on the proliferation, metabolism, and differentiation of rabbit mesenchymal stem cells. Cell Biol Toxicol 25(6):573-586

Colbert AP, Wahbeh H, Harling N, Connelly E, Schiffke HC, Forsten C, Gregory WL, Markov MS, Souder JJ, Elmer P, King V (2009) Static magnetic field therapy: a critical review of treatment parameters. Evid Based Complement Alternat Med 6(2):133-139

De Mattei M, Caruso A, Traina GC (1999) Correlation between pulsed electromagnetic fields exposure time and cell proliferation increase in human osteosarcoma cell lines and human normal osteoblast cells in vitro. Bioelectromagnetics 20(3):177-182

Del Bue M, Riccò S, Ramoni R, Conti V, Gnudi G, Grolli S (2008) Equine adipose-tissue derived mesenchymal stem cells and platelet concentrates: their association in vitro and in vivo. Vet Res Commun 32(1):51-55

Deregibus MC, Tetta C, Camussi G (2010) The dynamic stem cell microenvironment is orchestrated by microvesicle-mediated transfer of genetic information. Histol Histopathol 25:397-404

Esposito M, Lucariello A, Costanzo C, Fiumarella A, Giannini A, Riccardi G, Riccio I (2013) Differentiation of human umbilical cord-derived mesenchymal stem cells, WJ-MSCs, into chondrogenic cells in the presence of pulsed electromagnetic fields. In Vivo 27(4):495-500

Fierabracci A, Del Fattore A, Luciano R, Muraca M, Teti A, Muraca M (2013) Recent advances in mesenchymal stem cell immunomodulation. The role of microvesicles. Cell Transplant. doi:10.3727/096368913X675728

Ganesan K, Gengadharan AC, Balachandran C, Manohar BM, Puvanakrishnan R (2009) Low frequency pulsed electromagnetic field - a viable alternative therapy for arthritis. Indian J Exp Biol 47: 939-948

Gartzke J, Lange K (2002) Cellular target of weak magnetic fields: ionic conduction along actin filaments of microvilli. Am J Physiol Cell Physiol 283(5):1333-1346

Gimble JM, Katz AJ, Bunnell BA (2007) Adipose-derived stem cells for regenerative medicine. Circ Res 100(9):1249-1260

Godwin EE, Young NJ, Dudhia J, Beamish IC, Smith RK (2012) Implantation of bone marrow-derived mesenchymal stem cells demonstrates improved outcome in horses with overstrain injury of the superficial digital flexor tendon. Equine Vet J 44:25-32

Grassi C, D'Ascenzo M, Torsello A, Martinotti G, Wolf F, Cittadini A, Azzena GB (2004) Effects of $50 \mathrm{~Hz}$ electromagnetic fields on voltage-gated $\mathrm{Ca}^{2+}$ channels and their role in modulation of neuroendocrine cell proliferation and death. Cell Calcium 35(4):307-315

Grzesiak J, Marycz K, Czogala J, Wrzeszcz K, Nicpoń J (2011) Comparison of behavior, morphology and morphometry of equine and canine adipose derived mesenchymal stem cells in culture. Int J Morphol 29(3):1012-1017

Guest DJ, Smith MR, Allen WR (2008) Monitoring the fate of autologous and allogeneic mesenchymal progenitor cells injected into the superficial digital flexor tendon of horses: preliminary study. Equine Vet J 40:178-181

Heermeier K, Spanner M, Träger J, Gradinger R, Strauss PG, Kraus W, Schmidt J (1998) Effects of extremely low frequency electromagnetic field (EMF) on collagen type I mRNA expression and extracellular matrix synthesis of human osteoblastic cells. Bioelectromagnetics 19(4):222-231

Ikehara T, Yamaguchi H, Miyamoto H (1998) Effects of electromagnetic fields on membrane ion transport of cultured cells. J Med Invest 45(1-4):47-56

Inal JM, Kosgodage U, Azam S, Stratton D, Antwi-Baffour S, Lange S (2013) Blood/plasma secretome and microvesicles. Biochim Biophys Acta 1834(11):2317-2325

Kotani H, Kawaguchi H, Shimoaka T, Iwasaka M, Ueno S, Ozawa H, Nakamura K, Hoshi K (2002) Strong static magnetic field stimulates bone formation to a definite orientation in vitro and in vivo. J Bone Miner Res 17(10):1814-1821

Landry PS, Sadasivan KK, Marino AA, Albright JA (1997) Electromagnetic fields can affect osteogenesis by increasing the rate of differentiation. Clin Orthop Relat Res 338:262-270

Liu J, Huang J, Lin T, Zhang C, Yin X (2009) Cell-to-cell contact induces human adipose tissue-derived stromal cells to differentiate into urothelium-like cells in vitro. Biochem Biophys Res Commun 390(3):931-936

Majka M, Kijowski J, Lesko E, Goźdizk J, Zupanska B, Ratajczak MZ (2007) Evidence that platelet-derived microvesicles may transfer platelet-specific immunoreactive antigens to the surface of endothelial cells and CD34+ hematopoietic stem/progenitor cells - implication for the pathogenesis of immune thrombocytopenias. Folia Histochem Cytobiol 45(1):27-32

Maręziak M, Marycz K, Śmieszek A, Lewandowski D, Toker NY (2014) The influence of static magnetic fields on canine and equine mesenchymal stem cells derived from adipose tissue. In Vitro Cell Dev Biol Anim 50(6):562-571

Marion NW, Mao JJ (2006) Mesenchymal stem cells and tissue engineering. Methods Enzymol 420:339-361

Markov MS (2007) Therapeutic application of static magnetic fields. Environmentalist 27:457-463

Marycz K, Grzesiak J, Wrzeszcz K, Golonka P (2012a) Adipose stem cell combined with plasma-based implant bone tissue differentiation in vitro and in a horse with a phalanx digitalis distalis fracture: a case report. Vet Med 57(11):610-617

Marycz K, Toker NY, Grzesiak J, Wrzeszcz K, Golonka P (2012b) The therapeutic effect of autogenic adipose derived stem cells combined with autogenic platelet rich plasma in tendons disorders in horses in vitro and in vivo research. J Anim Vet Adv 11(23): $4324-4331$

Marycz K, Śmieszek A, Grzesiak J, Donesz-Sikorska A, Krzak-Roś J (2013) Application of bone marrow and adipose-derived mesenchymal stem cells for testing the biocompatibility of metal-based biomaterials functionalized with ascorbic acid. Biomed Mater 8(6): 065004. http://www.ncbi.nlm.nih.gov/pubmed/24280658

Mathivanan S, Fahner CJ, Reid GE, Simpson RJ (2012) ExoCarta 2012: database of exosomal proteins, RNA and lipids. Nucleic Acids Res 40(Database issue): $1241-1244$

Mizuno H, Tobita M, Uysal AC (2012) Concise review: adipose-derived stem cells as a novel tool for future regenerative medicine. Stem Cells 30(5):804-810

Muralidharan-Chari V, Clancy JW, Sedgwick A, D'Souza-Schorey C (2010) Microvesicles: mediators of extracellular communication during cancer progression. J Cell Sci 123(10):1603-1611

Nicpoń J, Marycz K, Grzesiak J (2013) Therapeutic effect of adiposederived mesenchymal stem cell injection in horses suffering from bone spavin. Pol J Vet Sci 16(4):753-754

Okano H, Onmori R, Tomita N, Ikada Y (2006) Effects of a moderateintensity static magnetic field on VEGF-A stimulated endothelial 
capillary tubule formation in vitro. Bioelectromagnetics 27(8):628640

Pesce M, Patruno A, Speranza L, Reale M (2013) Extremely low frequency electromagnetic field and wound healing: implication of cytokines as biological mediators. Eur Cytokine Netw 24(1):1-10

Qin K, Qiu LH, Zhong M, Wang ZY (2004) The effect of static magnetic field on bone morphogenetic protein-2 in periodontal membrane of the rat. Shanghai Kou Qiang Yi Xue 13(4):275-277

Qiu LH, Qin K, Zhong M, Wang ZY (2005) Effect of static magnetic field on bone morphogenetic protein-2 in periodontal membrane of experimental periodontitis rat. Hua Xi Kou Qiang Yi Xue Za Zhi 23(4):319-321

Qiu LH, Zhong M, Tang XN, Wang ZY (2007) Effect of static magnetic field on bone morphogenetic protein 2 and collagen type I of osteoblast cell. Shanghai Kou Qiang Yi Xue 16(1):33-35

Raisi A, Azizi S, Delirezh N, Heshmatian B, Farshid AA, Amini K (2014) The mesenchymal stem cell-derived microvesicles enhance sciatic nerve regeneration in rat: a novel approach in peripheral nerve cell therapy. J Trauma Acute Care Surg 76(4):991-997

Raposo G, Stoorvogel W (2013) Extracellular vesicles: exosomes, microvesicles, and friends. J Cell Biol 200(4):373-383

Ratajczak MZ (2011) The emerging role of microvesicles in cellular therapies for organ/tissue regeneration. Nephrol Dial Transplant 26(5):1453-1456

Rosen AD (2003) Mechanism of action of moderate-intensity static magnetic fields on biological systems. Cellular Biochem Biophys $39: 163-173$
Ross CL, Harrison BS (2013) The use of magnetic field for the reduction of inflammation: a review of the history and therapeutic results. Altern Ther Health Med 19(2):47-54

Roth V (2006) www.doubling-time.com/compute.php. Accessed 15 May 2014

Sabin K, Kikyo N (2014) Microvesicles as mediators of tissue regeneration. Transl Res 163(4):286-295

Stratton D, Lange S, Inal JM (2013) Pulsed extremely low-frequency magnetic fields stimulate microvesicle release from human monocytic leukaemia cells. Biochem Biophys Res Commun 430(2):470-475

Torbet J, Ronzière MC (1984) Magnetic alignment of collagen during self-assembly. Biochem J 219(3):1057-1059

Turturici G, Tinnirello R, Sconzo G, Geraci F (2014) Extracellular membrane vesicles as a mechanism of cell-to-cell communication: advantages and disadvantages. Am J Physiol Cell Physiol 306(7): C621-C633

Vergallo C, Dini L, Szamosvölgyi Z, Tenuzzo BA, Carata E, Panzarini E, László JF (2013) In vitro analysis of the anti-inflammatory effect of inhomogeneous static magnetic field-exposure on human macrophages and lymphocytes. PLoS One 8(8):e72374

Wang IE, Lu HH (2006) Role of cell-cell interactions on the regeneration of soft tissue-to-bone interface. Conf Proc IEEE Eng Med Biol Soc 1:783-786

Zomorodian E, Eslaminejad MB (2012) Mesenchymal stem cells as a potent cell source for bone regeneration. Stem Cells Int 2012(980353):1-9 